\title{
An Infrared Detection Method Used In Electrical Equipment Fault Diagnosis
}

\author{
Li Feng ${ }^{1}$, Li Jianfeng², Meng Yu², Tong Rui ${ }^{2}$, Liu Ren², Zu Bo², Liu Wei ${ }^{2}$ Liu Lin², \\ Wang Zhaoxia', Cheng Xingjun', Chen Guorui ${ }^{3}$, Liu Min² \\ ${ }^{1}$ Information and Communication Branch, Liaoning Electric Power Company Limited, State Grid, \\ China \\ ${ }^{2}$ Fushun Power Supply Company, Liaoning Electric Power Company Limited, State Grid, China \\ ${ }^{3}$ Liaoning Electric Power Company Limited, State Grid, China \\ fushunpowersupply@163.com
}

Keywords: Electrical equipment; Infrared; Temperature; Diagnosis; Accuracy

\begin{abstract}
In order to improve the fault detection and diagnosis infrared effect, describes the fault type infrared detection equipment; analysis of the factors affecting the accuracy of the temperature measurement; analysis approach proposed in addition to proper use, it also must be on a variety of factors that affect the test results adequately estimate and expected to find the temperature focused, analytical equipment thermal state changes, timely, accurate knowledge of the internal device, external heating, ensure safety equipment, stable operation.
\end{abstract}

\section{Introduction}

With the continuous development of power equipment fault diagnosis technology, modern infrared technology continues to mature and perfected, infrared detection and diagnosis technology of electrical equipment as a simple, efficient online monitoring technology equipment, electrical equipment is to ensure the safety, economic operation, to reduce preventive testing and inspection outage times, effectively prevent accidents, to provide a new method based on the state overhaul. How to further improve the accuracy of infrared detection, in addition to using the correct method of analysis, but also to deal with the various factors affecting the quality of the temperature study, understand and master the equipment fever principle, focused, timely and accurate discovery of device failure[1-2].

Type of fault detection of infrared temperature measurement technology electrical equipment can be divided into two categories: External thermal failure of equipment means equipment heating parts exposed to the environment, the use of infrared camera can be directly observed surface heat distribution state, and quickly determine its position. The main reason for failure of external heat is generated by the contact resistance equipment components caused by abnormal increase. If the contact surface is not flat, surface roughness or processing equipment connections and improper installation by the role of the external stress in operation, resulting in loose connections and part of aging and other reasons. Common equipment external faults are: all exposed parts of electrical connectors, such as isolation switch contacts, wall tube fittings, and electrical equipment outlet joints.

Electrical equipment failure due to internal heat, and heat sealed to the inside of the machine parts, can not be directly observed by thermal imager, to determine the specific location of the fault, the only heat distribution by acquiring the device surface for analysis, analogy be judged. Equipment internal electrical connections or contacts bad, caused by the current thermal effect, internal dielectric loss increases, the internal voltage distribution is bad, internal insulation aging, moisture, caused by lack of oil or vortex (iron loss increases) and so may cause internal equipment malfunction. 


\section{The measurement affecting factors analysis}

(1) Testing conditions

The accuracy of the temperature measurement is the basis of infrared equipment fault diagnosis, requiring accurately obtain the temperature distribution of the device under test or failure of the relevant parts of temperature and temperature rise. These data are judged equipment failure property, location and severity of an objective basis. Therefore, the calculation of the relevant parts of the test equipment failure and a reasonable temperature correction, the key will be to improve the accuracy of detection equipment surface temperature. However, when in the field of infrared detection equipment, test conditions and the impact due to changes in the environment, may lead to the same equipment due to different testing conditions, and get different results. Therefore, in order to improve the accuracy of infrared detectors must be on-site during the test on the test results of the analysis process, or, to take appropriate countermeasures and measure the results of the detection site for a reasonable correction or choose good testing conditions, such as in the morning day out before or after sunset detection to reduce the impact of external radiation detection equipment[3].

(2) Operating status

Electrical equipment faults proportional to the square of the current heating effect caused by a fault (conductive circuit fault), the heating power and the load current value; two voltage proportional to the heating effect caused by failure (dielectric breakdown), the heating power and operating voltage squared . Operating voltage and load current size of the device, will directly affect the effectiveness of infrared detection and fault diagnosis. If the load is low load operation or equipment failure will cause the fever is not obvious, even more serious fault exists, it is impossible to form a characteristic thermal anomalies exposed. Only when the device is running at rated voltage, and the greater the load, the heat and the temperature was more serious point of failure characteristic thermal anomalies also exposed the more obvious. Therefore, during an infrared detector, in order to obtain reliable test results, should be as selective detection at rated voltage and full load conditions. If conditions permit, you can make scheduling departments with equipment under continuous full load operation for some time, so that there is enough equipment fault location fever and ensure its surface temperature to stabilize, or just put into operation for the new technological transformation of equipment, particularly finished important. If you are unable to meet the full load operating conditions, should also be translated into the DUT temperature rise at rated current comprehensive judgments[4,5].

(3) Equipment surface emissivity

Infrared detection devices are electrical equipment by surface emissivity and infrared radiation power, to get the device temperature information. In the infrared detector receives infrared radiation power of the device under the same conditions, the emissivity of the surface of the device under test different test results obtained are also different. Due to surface emissivity of the object mainly depends on the material properties and the surface state (such as surface oxidation of the coating material, roughness and contamination state, etc.), in order to obtain accurate measurements, the subject must know the emissivity of the target value, correction parameters for the output value of the temperature measured emissivity correction. Two other countermeasures to eliminate emissivity influence on the test results are: When using infrared cameras to measure, the first piece of equipment to detect the emissivity value of the measured surface emissivity correction to obtain reliable temperature measurement results improve the reliability of detection; for hot Defect frequent equipment for the detection result with good comparability can paint applicator appropriate to increase the faulty equipment and stable surface emissivity value, in order to obtain the true surface of the target device temperature.

(4) Atmospheric attenuation

Since the surface of the target electrical equipment infrared radiation energy is transmitted through the atmosphere to the infrared detection instrument, the measurement accuracy will be attenuated atmospheric water vapor absorption, carbon dioxide and carbon monoxide gas molecules and particles suspended in the air scattering attenuation. The degree of attenuation of radiation energy equipment and testing instruments also measured the distance between the devices to have a 
relationship, the greater the distance, the more severe attenuation, equipment failure subjects with normal parts of the site radiation contrast is reduced, while the infrared instrument receives target energy value is reduced, so that the instrument displays the measured temperature is lower than the actual fault point temperature value, thereby resulting in missed or erroneous diagnosis. In particular, low temperature equipment inspected, it is negative. So when the infrared temperature measurement work going on, try to choose a relatively dry atmospheric environment, clean the season temperature measurement, without compromising security conditions shorten the detection distance as possible, in order to the actual measured temperature value.

Table 1 Reference materials commonly used emissivity

\begin{tabular}{|c|c|c|}
\hline Material & Temperature ${ }^{\circ} \mathrm{C}$ & Emissivity approximation \\
\hline Polished aluminum & 100 & 0.09 \\
\hline Mild alumina & $25-600$ & $0.10-0.20$ \\
\hline Polished cast iron & 200 & 0.21 \\
\hline Machining cast iron & 20 & 0.44 \\
\hline Completely rusted cast iron & $40-250$ & 0.95 \\
\hline Insulation sheet & - & $0.91-0.94$ \\
\hline Porcelain (bright) & 23 & $0.90-0.92$ \\
\hline
\end{tabular}

(5) Background radiation

During outdoor power equipment infrared detection, infrared radiation detection equipment received includes not only the subjects of radiation emitted by the corresponding parts of the device, but also in other parts of the reflection, including equipment and backgrounds, as well as direct income solar radiation. These emissions are measured temperature of the device will cause interference to the site, the impact test results. For example: On a clear day to detect, especially in the vicinity of the main run of the change, the background is a large area of sunlight radiation and other hot objects, make additional measurements have a greater temperature rise, the additional temperature rise and the ambient temperature and degree sunny weather related. These factors not only affect the temperature distribution of the device point of failure, and even weak target thermal image caused by undetected. In order to reduce the environmental impact of background radiation, should take the following countermeasures:

In addition, you can take shelter in the detection of the appropriate measures, or the installation of the appropriate infrared filters on infrared imaging device to filter out the sun and other background radiation.

\section{Infrared detection method}

(1) Thermal imaging characteristics Criterion

All electrical equipment, in the absence of any fault conditions can be obtained by surface heat distribution or infrared thermographs feature maps under normal operating conditions. Once the electrical device has an internal or external fault, then the fault by the internal member and the heat transfer medium, or other forms of heat exchange, the faulty portion of the stabilizing device surface temperature or temperature distribution will change. Therefore, the characteristics and the size of the thermal field by changing the distribution of temperature rise, we can make an accurate diagnosis of the property, fault location and severity of the problem.

(2) Comparing the phases value

Three-phase high voltage electrical equipment mostly run, under normal circumstances, the voltage applied to each phase or three-phase current through substantially the same. In other words, the same part of each phase of the device should be as normal and stable temperature. So between the three phases in the same group with a comparable device, similar equipment at the same time in the same place and the same power role is comparable to each other. While the probability of the same part of the three-phase fault occurs while the same is small, for this reason, the diagnosis may be the same part of the temperature rise of between three-phase horizontal comparison. If an electrical device a, b, c three-phase exist any more than about $10{ }^{\circ} \mathrm{C}$ temperature difference between the two parts of the same phase, you can put a higher temperature phase of the parts of the 
initial diagnosis of faults. As shown in Figure 1.

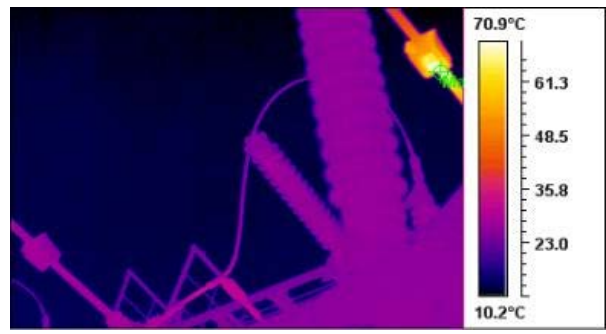

Figure $1 \mathrm{~B} 、 \mathrm{C}$ phase knife temperature difference

(3) Compared with discrimination law

Electrical equipment one phase load current flowing through the same, if the same occurs with significant temperature difference between the different parts, it often indicates the presence of anomalies or defects at a higher temperature. For example, in high-voltage transmission line connector in, if there is significant rise compared with the connection with a phase conductor temperature meters away, it means that the connector poor connection. As shown in Figure 2.

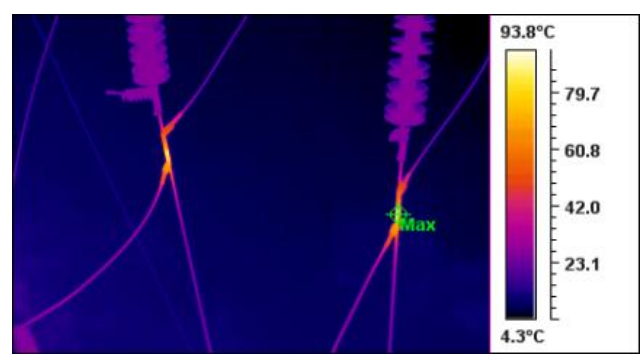

Figure 2 Poor connection member due to heat generation

\section{Conclusion}

In order to better improve the accuracy of infrared temperature measurement technology to ensure continuous and reliable operation of the grid, through the accumulation of a variety of equipment failure characteristics, patterns of research and practice, and improve the accuracy of diagnosis and qualitative judgment, safety equipment run lay the technical foundation.

\section{References}

[1] Hu Hongguang. Power infrared diagnostic technology operations and management. China Electric Power Press, 2006

[2] SETCs. Live equipment Infrared Diagnostic Technique Guidelines， 2005

[3] Zhangheng. Electrical equipment fault diagnosis IR. China Electric Power Press, 1999

[4] Shanghai Electric Power Company. Infrared Power Equipment 101 cases diagnosed, China Electric Power Press, 2003

[5] Dongqiguo. Power transformer fault and diagnosis. China Electric Power Press, 2000. 